\title{
Upper urinary tract inverted papillomas: Report of 10 cases
}

\author{
JIN-DAN LUO, PING WANG, JUN CHEN, BEN LIU, SHUO WANG, BO-HUA SHEN and LI-PING XIE
}

Department of Urology, The First Affiliated Hospital, School of Medicine, Zhejiang University, Hangzhou, Zhejiang 310003, P.R. China

Received February 21, 2012; Accepted April 20, 2012

DOI: $10.3892 / \mathrm{ol} .2012 .706$

\begin{abstract}
The aim of the study was to review the clinical features and treatments of 10 (9 males and 1 female; age range, 61-73 years; median age, 67 years) upper urinary tract inverted papilloma (IP) cases between 1995 and 2010. The clinical syndromes, diagnostic procedures, treatments and results of the follow-up were evaluated. The results showed that the site of tumor development was the ureter in 6 cases and the renal pelvis in 4 cases. It was also identified that 7 tumors developed on the left side and 3 developed on the right side of the ureter and renal pelvis, respectively. A nephroureterectomy was performed in the first 6 cases, while a partial ureterectomy was performed in 3 cases and a local resection was performed endoscopically in 1 case. All but 2 tumors were solitary, ranging from 5 to $30 \mathrm{~mm}$ in diameter. Occurrence in association with transitional cell carcinoma was identified in one case. All 10 patients were subject to follow-up (range, 19-120 months; median, 59 months), during which no recurrence was found. Local excision is considered as adequate treatment when upper urinary tract IP is diagnosed according to strictly defined criteria.
\end{abstract}

\section{Introduction}

Inverted papilloma (IP) is a rare lesion of the urinary tract system, which was first described in 1927 as adenomas in the bladder (1) before it was described in the 1960s as IP (2). IP is a tumor formed by proliferating urothelium arranged as inverting cords, which nest in continuity with an overlying intact urothelium. They account for approximately $2.2 \%$ of all urothelial neoplasms (3) and the majority of IP cases occur in the bladder, while upper urinary tract IP cases are extremely rare. IP tumors are generally regarded as benign lesions, however, certain controversies remain. At present, few studies have reported the IP occurrence in the upper urinary tract. The present study collected 10 upper urinary tract IP cases treated

Correspondence to: Dr Li-Ping Xie, Department of Urology, The First Affiliated Hospital, School of Medicine, Zhejiang University, Hangzhou, Zhejiang 310003, P.R. China

E-mail: lipingxie_2011@163.com

Key words: upper urinary tract, inverted papilloma, prognosis, excision at The First Affiliated Hospital of Zhejiang University, China, and reviewed the clinical syndromes, diagnostic procedures, treatment approaches and follow-up study results of each patient.

\section{Patients and methods}

The present study included 10 patients who had been hospitalized in our Department of Urology between 1995 and 2010 as a result of IP within the upper urinary tract. Of the 10 patients, 9 were male and 1 was female (age range, 61-73 years; median, 67 years). The evaluations included personal data, medical history, symptoms, localization of the disease, recurrences and malignant transformations. No patient in this study had a history of urothelial carcinoma; however, 5 patients had a history of smoking. In all cases, an intravenous urogram (IVU) and a computed tomography (CT) scan were performed. Two patients underwent ureteroscopic evaluation, and biopsies of the lesions were shown to be consistent with IP. All 10 patients were subject to either a nephroureterectomy, partial ureterectomy or local resection. Subsequently, the patients were scheduled for close follow-up (range, 19-120 months; median, 59 months) with ultrasonic scanning of the urinary tract and cystoscopy. This study was approved by the Clinical Research Ethics Committee at the First Affiliated Hospital of Zhejiang University and written consent was obtained from all patients.

\section{Results}

The clinical features and treatment approaches of each patient are summarized in Table I. The initial symptoms of the disease included gross hematuria and flank pain (on health examination), which were observed in $6(60 \%)$ and $2(20 \%)$ cases, respectively. No symptoms were observed in 2 (20\%) cases. The site of development was the ureter in 6 cases $(60 \%)$ and the renal pelvis in 4 cases $(40 \%)$. In addition to this, 7 cases (70\%) occurred on the left side and 3 cases $(30 \%)$ on the right side of the ureter and renal pelvis, respectively. Following IVU and CT examination, a filling defect was observed in all 10 patients, with hydronephrosis observed in 9 cases and a kidney stone observed in 1 case. Retrograde ureter pyelography confirmed the filling defects in 3 cases and negative urine cytology results were obtained in 3 cases.

All 10 patients underwent a surgical procedure. A nephroureterectomy was performed in 6 patients, a partial ureterectomy was performed in 3 patients with IP in the ureter, 
Table I. Clinical characteristics of 10 patients with IP.

\begin{tabular}{rcllllll}
\hline Patient & Age (years) & Gender & \multicolumn{1}{c}{ Location } & Chief complaint & Multiplicity & \multicolumn{1}{c}{ Treatment } & Recurrence \\
\hline 1 & 62 & Male & Right renal pelvis & Asymptomatic & Single & Nephroureterectomy & None \\
2 & 66 & Male & Left renal pelvis & Hematuria & Single & Nephroureterectomy & None \\
3 & 70 & Male & Right ureter & Hematuria & Single & Nephroureterectomy & None \\
4 & 64 & Male & Left renal pelvis & Hematuria & Single & Nephroureterectomy & None \\
5 & 61 & Male & Left ureter & Loin pain & Single & Partial ureterectomy & None \\
6 & 67 & Male & Left ureter & Asymptomatic & Multiple & Nephroureterectomy & None \\
7 & 67 & Male & Left ureter & Hematuria & Multiple & Local resection & None \\
8 & 73 & Male & Left ureter & Hematuria & Single & Partial ureterectomy & None \\
9 & 73 & Female & Right renal pelvis & Loin pain & Single & Nephroureterectomy & None \\
10 & 68 & Male & Left ureter & Hematuria & Single & Partial ureterectomy & None \\
\hline
\end{tabular}

IP, inverted papilloma.

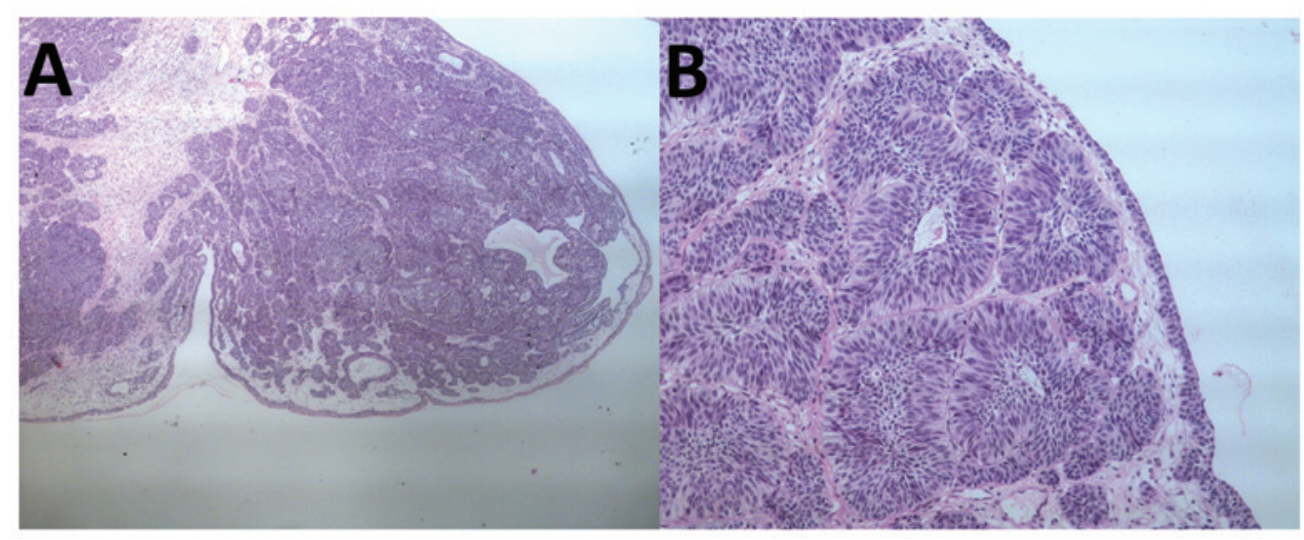

Figure 1. (A) Histological appearance of an IP demonstrating intact surface lining urothelium and underlying thin anastomosing urothelial islands (H\&E staining; magnification, x50). (B) Anastomosing cords of bland transitional cells with specific small gland-like areas (H\&E staining; magnification, x200). IP, inverted papilloma.

where two patients had a positive biopsy for IP preoperatively. A local resection of a nodular polypoid lesion protruding in the lumen of the ureter, was performed in 1 patient endoscopically using a holmium laser (Table I). The pathological examination of the frozen IP cell sections confirmed the diagnosis of IP. In one case, association with transitional cell carcinoma (TCC) (Grade 1, stage Ta) was found adjacent to the IP. In this case, a partial ureterectomy was performed since the renal function on the contralateral side appeared to be interrupted from IVU results.

All but 2 tumors were solitary, ranging from 5 to $30 \mathrm{~mm}$ in diameter. The frozen sections demonstrated a pedunculated nodule arising from the transitional cell epithelium. The tumor was found to be covered by normal urothelium and the tumor cells only demonstrated a slight degree of nuclear polymorphism. There was an endophytic proliferation of transitional cells arranged in broad cords and islands. The cells at the periphery of certain islands demonstrated a tendency to palisade, and few central gland-like spaces were present. No significant atypia or mitotic activity was noted. The typical histological appearances of IP cells are shown in Fig. 1. During the follow-up period, no recurrence of IP or subsequent TCC was observed.

\section{Discussion}

IP is a rare tumor that develops within the bladder in $90 \%$ of cases (4). It is defined by its distinctive gross and microscopic appearance, as well as its benign clinical course, characterised by a lack of invasive growth and metastases, low incidence of multiplicity and low incidence of local recurrence. IP of the upper urinary tract is extremely uncommon as the majority of cases develop within the trigone of the bladder, neck or prostatic urethra. However, the occurrence of upper urinary tract localization is not surprising when considering how the urothelial-lined tissue behaves as a single pathophysiological unit. It is usually found in patients within the 6th or 7th decade of life, although a wide age range (26 to 85 years) (1) and a mean age of 67 years was observed in our patients. Upper urinary tract IP has been demonstrated to develop more in 
males than females, with a male:female ratio of 9:1 (1). The same results were obtained from our cases. Usually, the IP lesion occurs as a solitary lesion, although 3.6-6\% appear to be bilateral or multicentric (5). IP lesions are twice as common in the ureter in comparison to the renal pelvis (6) and the lesions range from approximately 5 to $30 \mathrm{~mm}$ up to $3-4 \mathrm{~cm}$ in diameter (7).

The associated clinical symptoms of upper urinary tract IP do not differ from those in other urothelial neoplasias, with hematuria and renal colic being the most common clinical manifestations of all upper urinary tract lesions. In the present study, 6 patients $(60 \%)$ and 2 patients $(20 \%)$ complained primarily of gross hematuria and loin pain, respectively. However, this disease can also be asymptomatic, which was demonstrated in 2 of our patients, and may be diagnosed during unrelated clinical investigations. Preoperative diagnosis of IP is difficult. An IVU is used to identify filling defects or signs of obstruction, however, these findings are non-specific. Due to the intact layer of histologically normal urothelium that covers the IP lesion, it is not surprising that the cytological morphology falls within the normal or mild atypia range. In a literature review regarding IP, it was reported that urine cytology was specified in $8 \%$ of cases (22 of 277) (8). In our experience, cytology does not appear to be useful in diagnosis and an accurate diagnosis requires a biopsy and visualization through endoscopic examination, which we consider is more sensitive than indirect radiographic studies. In this study, 2 patients underwent ureteroscopic evaluation and biopsies made the nature of the lesions evident preoperatively. With the development of flexible ureteropyeloscopy, it is now possible to examine the entire upper urinary tract. Certain authors have noted that IP lesions have a gross appearance described as 'broad stalked', 'more solid' or 'less papillary' in comparison to transitional papillary tumors. The endoscopic procedure with biopsy provides a preoperative diagnosis and therapeutic indications, which are able to free the patient from unnecessary nephroureterectomy.

Treatment of upper urinary tract IP remains controversial. Various surgical procedures are used, such as total nephroureterectomy, partial resection of the ureter and endoscopic surgery. In the past, numerous cases have been treated aggressively with nephroureterectomy, which was performed in $60 \%$ of the cases in this study. With the development of endoscopy, endoscopical local excision of ureteral IP is considered adequate treatment by certain experts (9), as well as partial resection of the ureter, providing that the IP is positively diagnosed prior to or during surgery. However, differentiating ureteral IP from urothelial carcinoma and coexistence of malignancy, still complicate the preoperative diagnosis. Laparoscopy is also a minimally invasive approach in comparison with open surgery. It maintains the ability to obtain pathological diagnosis and intraoperative assessment and provides a definitive treatment, such as primary excision, segmental ureterectomy or nephroureterectomy.

The etiology of IP is not yet clearly understood. Several studies have argued the importance of inflammatory causative factors, which is further supported by its close histological resemblance to cystitis cystica, proliferative cystitis and glandularis (10). Sung et al also suggested that the correlation between IP and smoking required further investigation (11). In their study, $61 \%$ (28 of 46) of patients, had a history of smoking (11). In our study, 50\% (5 of 10) of patients had a history of smoking. Kunze et al subdivided IP into two morphologically distinct types. Firstly, a glandular type, which is composed of nests of urothelium with either pseudoglandular spaces or true glandular elements containing mucicarminophilic secretions and mucous-secreting cells. Secondly, a trabecular type, which is composed of anastomosing cords and trabeculae of urothelial cells invaginating the lamina propria (4). Although marked cytological atypia favors a diagnosis of inverted urothelial carcinoma, focal mild cytological atypia is considered acceptable in IP. Broussard reported that cases harboring focal cytologic atypia (less than $5 \%$ ) did not demonstrate significant cell proliferation characteristics, and there was no tumor recurrence or progression to urothelial carcinoma found during the follow-up (12).

A debate over the appropriate classification of IP as either a true benign neoplasm or a urinary malignant precursor lesion, has existed since its first description. The histological appearance, its rare multiplicity (observed in 15 of $277,5.4 \%$, of patients) and its low recurrence rate (approximately 1-7\% of cases), provide evidence to favor the benign classification of IP. Certain authors have concluded that IP does not appear to be a risk factor for TCC and to the best of our knowledge, there are no reported cases of invasion or metastasis by IP. However, sporadic cases of IP with concurrent urothelial carcinoma or malignant features, have also been recently documented, rendering the clinical image of this entity controversial. Cheng et al have reported that the incidence of associated synchronous urothelial carcinoma and subsequent urothelial carcinoma in the lower urinary tract IP was 6 and 3\%, respectively (13). More notably, in a group of 73 patients, $16(22 \%)$ appeared to have concomitant previous TCC (8). Therefore, there is a strong association of IP and urothelial malignancy in the upper tract. The frequency of synchronous malignancy in ureteric IP was reported to be three times the frequency of that found in similar lesions in the bladder. Spevack et al have reported that 7 of $30(23 \%)$ cases of upper urinary tract IP were complicated with TCC at a different location or time (14). Complication with TCC was also found in 1 patient in this study. Concordance with or a history of TCC in patients with an IP suggests that the two types of tumors share certain causative factors. It was also recommended that the possibility of TCC complication should be taken into consideration when the lesion in the upper urinary tract is larger than $20 \mathrm{~mm}$, even with a diagnosis made by biopsy prior to and during surgery. Therefore, there is a correlation between IP and TCC to some extent, although IP is generally considered to be a benign lesion. The malignant potential of IP lesions remain ambiguous due to the unknown etiology and low incidence rate.

Since the recognition of IP as a distinct lesion of the urinary tract, clinical follow-up appears to be necessary following excision, despite the uncertainty regarding the method and length. Certain authors have argued that surveillance protocols, as rigorous as those employed in the management of urothelial carcinoma, appear unnecessary for this benign entity due to its low incidence of recurrence and markedly favorable prognosis during follow-up $(8,11)$. However, it is accepted by the majority of authors that the patients should be followed with endoscopy and radiographic studies similar to those observed in patients 
with low-grade TCC (15), particularly in upper urinary tract IP cases. As a surveillance protocol of the upper urinary tract IP, we recommend cystoscopy and ultrasonic scans every 6 months for the first 2 years, and then annually. This is due to the fact that the time to recurrence in the majority of cases is no more than 2 years following surgery.

In conclusion, IP of the upper urinary tract is a rare and benign lesion. The 10 cases presented in this study support previous findings regarding male predominance, multiplicity and difficulty with preoperative diagnosis. We have reason to consider that certain cases of the upper urinary tract IP are able to recur and have a malignant potential. The finding of an IP in any part of the urinary tract should alert the urologist to conduct an investigation of the entire urinary tract. They should also emphasize to the patient the necessity for close clinical follow-up, particularly when concurrent TCC presents.

\section{References}

1. Paschkis R: Über Adenoma der Harnblase. Z Urol Chir 21: 315-325, 1927 (In German).

2. Potts IF and Hirst E: Inverted papilloma of the bladder. J Urol 90: 175-179, 1963.

3. Isaac J, Lowichik A, Cartwright P and Rohr R: Inverted papilloma of the urinary bladder in children: case report and review of prognostic significance and biological potential behavior. J Pediatr Surg 35: 1514-1516, 2000.

4. Kunze E, Schauer A and Schmitt M: Histology and histogenesis of two different types of inverted urothelial papillomas. Cancer 51: 348-358, 1983.
5. Rozanski TA: Inverted papilloma: an unusual recurrent, multiple and multifocal lesion. J Urol 155: 1391, 1996.

6. De Knijff DW, Theunissen PH and Delaere KP: Inverted papilloma of the ureter with subsequent invasive bladder cancer. Acta Urol Belg 65: 45-46, 1997.

7. Kyriakos M and Royce RK: Multiple simultaneous inverted papillomas of the upper urinary tract. A case report with a review of ureteral and renal pelvic inverted papillomas. Cancer 63: 368-380, 1989

8. Witjes JA, van Balken MR and van de Kaa CA: The prognostic value of a primary inverted papilloma of the urinary tract. J Urol 158: 1500-1505, 1997.

9. Bagley DH, McCue P and Blackstone AS: Inverted papilloma of renal pelvis: flexible ureteroscopic diagnosis and treatment. Urology 36: 336-338, 1990.

10. Chiura AN, Wirtschafter A and Bagley DH: Upper urinary tract inverted papillomas. Urology 52: 514-516, 1998.

11. Sung MT, Maclennan GT, Lopez-Beltran A, Montironi R and Cheng L: Natural history of urothelial inverted papilloma. Cancer 107: 2622-2627, 2006.

12. Broussard JN, Tan PH and Epstein JI: Atypia in inverted urothelial papillomas: pathology and prognostic significance. Hum Pathol 35: 1499-1504, 2004.

13. Cheng CW, Chan LW, Chan CK, Ng CF, Cheung HY, Chan SY, Wong WS and To KF: Is surveillance necessary for inverted papilloma in the urinary bladder and urethra? ANZ J Surg 75: 213-217, 2005.

14. Spevack L, Herschorn S and Srigley J: Inverted papilloma of the upper urinary tract. J Urol 153: 1202-1204, 1995.

15. Kilciler M, Bedir S, Erdemir F, Ors O, Kibar Y and Dayanc M: Evaluation of urinary inverted papillomas: a report of 13 cases and literature review. Kaohsiung J Med Sci 24: 25-30, 2008. 